\title{
Inducción de ovulación: II pacientes clomifeno negativas
}

\author{
Gustavo Gómez Tabarez*; Luz Stella Gómez Mesa**
}

\begin{abstract}
RESUMEN: En pacientes que no responden a la terapia con citrato de clomifeno, se utiliza un protocolo basado en dosis bajas de clomifeno y gonadotrofina menopáusica humana (HMG).

Se obtiene $51.2 \%$ de ovulación en 35 ciclos. Las pacientes obesas tienen una baja respuesta al esquema de inducción de ovulación lo mismo que las pacientes con una relación LH/FSH mayor de 2.68. No se presentan síntomas de hiperestimulación ovárica. En las pacientes que ovulan se utiliza $50 \mathrm{mg} /$ día por 5 dosis de clomifeno y un promedio de 2.2 ampollas de 75 UI de HMG por ciclo en promedio.
\end{abstract}

El promedio del tamaño del folículo al momento de ovulación es de $18.66 \pm 4.9 \mathrm{~mm}$. El $60 \%$ de las pacientes ovulan $24-48$ horas posterior a la aplicación de HCG; restante lo hacen a las 24 horas o menos.

Se discute la utilidad y las indicaciones de protocolos alternos en pacientes «resistentes» al clomifeno.

SUMMARY: We report the use of a combined teraphy of clomiphene citrate and human menopausal gonadotrophins (HMG) in «clomiphene resistant» patients to the usual protocol of up $150 \mathrm{mg} /$ day by 5 days. Using together low doses of clomiphene citrate and HMG we did reach $51.2 \%$ of ovulation in 35 cycles. Obesity and high relationship of LH/FSH resulted in low rates of ovulation. There was not simptoms of hyperstimulation syndrome. The average of preovulatory follicle diameter was $18,66 \pm 4.9 \mathrm{~mm} .60 \%$ of the ovulation occurred 24-48 hours after HCG aplication and the other $40 \%$ between the first 24 hours. We consider the above protocol is an option for use in patients clomiphene negative.

La falta de ovulación es causa de infertilidad en el $21 \%$ de las pacientes que consultan a la Sección de Endocrinología e infertilidad del departamento de Obstetricia y Ginecología. El resto se distribuye así: factor masculino $25 \%$; tuboperitoneal $46 \%$ cervical $4 \%$ y de causa desconocida el resto.

La tasa de ovulación presentada por nosotros con el uso de clomifeno es del orden del $58.8 \%$ (1).

El citrato de clomifeno es un medicamento pionero en inducción de ovulación (11). Algunas pacientes no logran ovulación con el uso continuado del mismo. Consideramos clomifeno negativas, aquellas pacientes que no responden a los esquemas tradicionales de este medicamento.

En este trabajo se utilizó como alternativa un esquema de Clomifeno y Gonadotrofina Menopáusica Humana en bajas dosis, en pacientes «resistentes» al clomifeno.

\section{Pacientes y métodos}

Se incluyeron 15 pacientes en un estudio clínico prospectivo de casos de pacientes que no respondieron a la inducción de ovulación con clomifeno.

Las pacientes consultaron a la Clínica de Infertilidad del Hospital Universitario del Valle (HUV) en un período de seis meses.

* Profesor Titular. Jefe Sección Endocrinología e Infertilidad. Departamento Obstetricia y Ginecología. Universidad del Valle.

** Bacterióloga y Laboratorista Clínica. Laboratorio Endocrinología e Infertilidad. Hospital Universitario del Valle.
La causa de consulta fue primariamente por infertilidad. Siguiendo el protocolo establecido para el estudio de las parejas infértiles se realizaron los siguientes exámenes y procedimientos básicos: espermograma, curva de temperatura basal (CTB), test post coito, laparoscopia diagnóstica y biopsia de endometrio. De acuerdo a la historia clínica se realizan pruebas complementarias como determinaciones hormonales específicas, test de penetración espermática, anticuerpos antiespermatozoides e histerosalpingografía (HSG).

Las pacientes objetos del estudio, se catalogaron como anovulatorias. Algunas tenían otro(s) factores comprometidos.

En el esquema de inducción de ovulación con clomifeno se inicia con $50 \mathrm{mgs}$./día del 5-9 del ciclo. Cuando el folículo alcanza $18 \mathrm{~mm}$ de diámetro se aplica 5000 UI de HCG.

Si no hay ovulación se repite el ciclo con 100 mgs y 150 mgs de clomifen en el segundo y tercer ciclo respectivamente. Consideramos «clomifeno negativas» aquellas pacientes que siguiendo el anterior esquema, no ovularon con $150 \mathrm{mgs} /$ día. Este fue el único criterio de inclusión al estudio.

La ovulación se determinó por medio de curva de temperatura basal (CTB) y seguimiento ecográfico del crecimiento folicular. El diagnóstico de ovulación por ecografía se hizo ante la desaparición de un folículo en crecimiento, la aparición de signos de cuerpo lúteo (CL), la presencia de líquido en el fondo de saco de Douglas y el grosor del endometrio.

En todas las pacientes se hicieron determinaciones de FSH, LH, PRL y T en el laboratorio central del hospital. 


\section{Protocolo de inducción de ovulación}

Se administran $50 \mathrm{mg} /$ día de clomifeno entre los días 59 del ciclo. El día 10 del ciclo se administra por vía IM. dos (2) ampollas de gonadotropina menopáusica humana (HMG) (Pergonal)* de 75 IU cada una y luego 2 ampollas día de por medio hasta que el folículo alcance un diámetro igual o mayor de $16 \mathrm{~mm}$. Cuarenta y ocho horas posterior a la última dosis de HMG, se aplica IM 5000 IU de gonadotrofina coriónica humana (HCG) (Profasi)*.

En casos de no haber crecimiento folicular 48 horas después de aplicar una dosis de 150 UI de HMG, se incrementa la dosis en 75 IU ( 1 ampolla). Si persiste sin crecimiento, se abandona el ciclo.

Seguimiento ecográfico. Se practica una ecografía el día 5 del ciclo y otra el día 9 del ciclo, último día del clomifeno. Se continúan realizando ecografías diarias hasta que el paciente presenta signos ecográficos de ovulación o hasta que se abandona el ciclo por no ovulación.

Ante una falla menstrual se determina $B$ HCG y si ésta es positiva se practica una ecografía transvaginal entre la cuarta y quinta semanas, para confirmar embarazo intrauterino. $\mathrm{Si}$ la prueba es negativa, se administran $10 \mathrm{mg}$ de acetato de medroxiprogesterona por 5 días y una vez se presenta la menstruación se reinicia el protocolo.

Se fue muy cuidadoso en la busca de signos y síntomas de hiperestimulación ovárica.

\section{Resultados}

Se relacionó el número de pacientes obesas $(120 \%$ de exceso sobre el peso ideal para la talla) y no obesas. En todos los pacientes se calculó la relación LH/FSH.

En los ciclos estudiados se determinó el porcentaje de ovulación, su relación con la obesidad y con la relación LH/ FSH.

Se hizo promedio del crecimiento folicular por día en los grupos ovulatorios y no ovulatorios. También el promedio del tamaño folicular al momento de ovulación. Tiempo entre última dosis de HMG y ovulación y tiempo entre dosis de HCG y ovulación.

Se indujeron 35 ciclos en las 15 pacientes.

El $46.6 \%$ de las pacientes $(7 / 15)$ fueron obesas.

En los 35 ciclos de estudio, 15 fueron en las pacientes obesas y 20 en las no obesas.

Se presentó ovulación en el $51.4 \%$ de los ciclos tratados. $10 / 15(66.6 \%)$ de las pacientes tuvieron una relación LH/ FSH $>$ de 2.68 .

El $57.1 \%(4 / 7)$ de las obesas y $75 \%$ (6/8) de las no obesas tenían una LH/FSH alterada. Figura 1.

$\mathrm{La} \mathrm{LH/FSH} \mathrm{en} \mathrm{las} \mathrm{pacientes} \mathrm{que} \mathrm{ovularon} \mathrm{fue} \mathrm{alterada} \mathrm{(>}$ $2.68)$ en el $42.8 \%(3 / 7)$ en las pacientes ovulatoria y $87.5 \%$ (7/8) en las pacientes que no ovularon. Figura 2.

En los 35 ciclos, las obesas ovularon en el $40 \%$ de sus ciclos y las no obesas en el $65 \%$. Figura 3.

El promedio de crecimiento folicular ( $\mathrm{mm} /$ día), en las pacientes ovulatorias fue de $2.6 \pm 1.4$ (1 desviación estandard, $\mathrm{DE})$ y las no ovulatorias de $0.44 \pm 0.29$ (1 DE). El folículo no muestra crecimiento durante los días de toma del clomifeno.

El tamaño del folículo al momento de la aplicación de HCG es de $18.66 \pm 4.9,(1 \mathrm{DE})$ cuarenta y ocho horas después de la última dosis de HMG, cuando el folículo invariablemente había quedado en alrededor de $16 \mathrm{~mm}$.

Figura 1

LH/FSH Y OBESIDAD

PACIENTES RESISTENTES AL CLOMIFENO

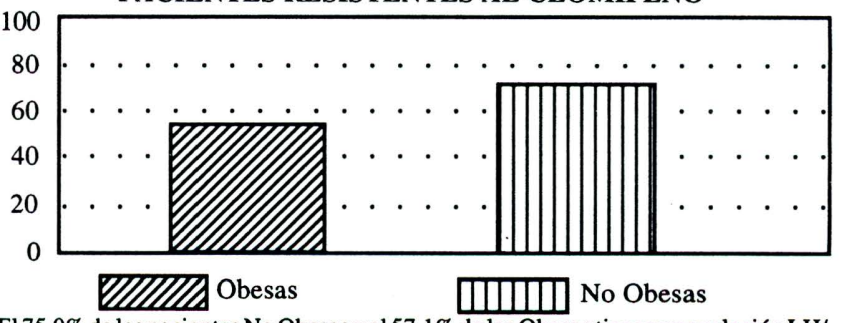

El 75.0\% de las pacientes No Obesas y el $57.1 \%$ de las Obesas tienen una relación LH/ FSH $>$ de 2.68

Figura 2

LH/FSH Y OVULACION

PACIENTES RESISTENTES AL CLOMIFENO

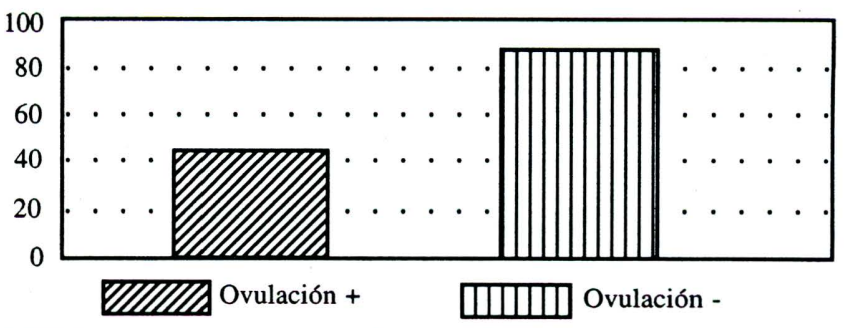

El $87.5 \%$ de las pacientes que no ovulan y el $\mathbf{4 2 . 8}$ que lo hacen tienen una relación LH/FSH $>$ de 2.68

Figura 3

OVULACION Y OBESIDAD

PACIENTES RESISTENTES AL CLOMIFENO

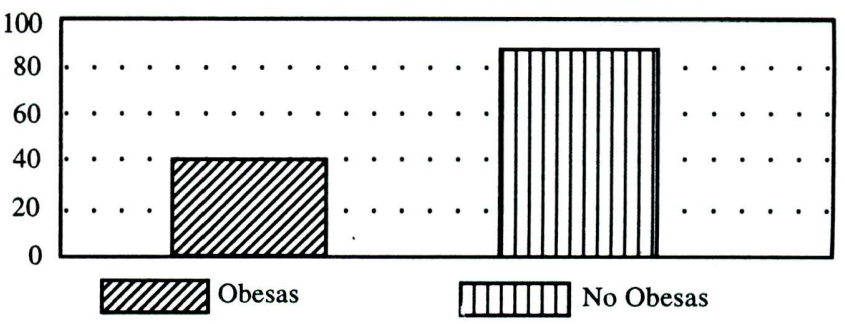

Porcentaje de ovulación en pacientes Obesas y No Obesas

El 60\% de los pacientes ovulan entre 24-48 horas después de la aplicación de la dosis de HCG. El otro $40 \%$ lo hacen en las primeras 24 horas.

No hubo ninguna paciente con síntomas ni leves de hiperestimulación ovárica en los pacientes objeto del presente estudio.

\section{Discusión y conclusiones}

Se han establecido innumerables esquemas de inducción de ovulación y los medicamentos inductores de ovulación crece cada vez más. Los más usados son el citrato de clomifeno y la gonadotrofina menopáusica humana (HMG), solos o en conjunto con gonadotrofina cariónica humana (hCG) (1213). Hay esquemas con FSH pura, y análogos de GnRh. También hay múltiples combinaciones de estos inductores $(2,4-5,7)$. Muchos de estos esquemas son alternativas a los pacientes resistentes al clomifeno (1-2, 4-5). Sin embargo, la mayoría son costosas y HMG, a dosis tradicionales, debe ser 
administrada con una monitoría cuidadosa, debido al peligro de hiperestimulación ovárica (14). Teóricamente al menos, la combinación de dos medicamentos determinaría una acción sinérgica, en sus efectos como inductor así como en sus efectos secundarios. Por lo tanto parece lógico que las mezclas de medicamentos se utilicen en dosis más bajas cada uno de sus componentes y con ello evitar los efectos indeseables propios de cada uno de ellos.

El incremento de las dosis citrato de clomifeno disminuye su efecto benéfico en cuanto a fertilidad se refiere, posiblemente por el aumento de su efecto antiestrogénico sobre endometrio, y moco cervical $(1,12)$, además altera el crecimiento folicular (3) y la esteroidogénesis ovárica (8).

El aumento de LH endógena se considera responsable de la dificultad de inducción ovulatoria en algunos pacientes. Los niveles altos de LH también se encuentran como explicación de la alta incidencia de abortos en pacientes con SOA e inducción de ovulación (6), debido a una hiperrespuesta a la acción de GnRh en estos pacientes (7).

Algunas observaciones indican que cuando se adopta un régimen de dosis bajas de gonadotropinas, la $\mathrm{LH}$ exógena tiene poco impacto en los niveles de LH endógena, lo que favorece la inducción de ovulación en pacientes anovulatorios crónicos con relaciones LH/FSH altos (7).

La HMG es costosa y como efecto secundario puede presentar hiperestimulación ovárica, que en el caso de ser grave, atenta contra la vida de la paciente. Sin embargo se ha documentado que aún sin el uso de niveles de estrógenos y teniendo en cuenta el tamaño folicular por ecografía confiable, se puede prevenir y evitar esta complicación además de que se puede predecir la ovulación (10).

En el caso de los pacientes clomifeno negativos, se busca una combinación, en el caso de nuestro estudio, con HMG a dosis bajas, para lograr mayor potencia estimulante de la ovulación, disminución de complicaciones (antiestrogenismo, hiperestimulación) y reducción de costos.

Con el protocolo establecido para inducción de ovulación en pacientes resistentes o clomifenonegativas se alcanzó éxito en la inducción de ovulación en el $51.42 \%$ de los ciclos tratados.

En todas nuestras pacientes utilizamos $50 \mathrm{mgs} /$ día de citrato de clomifeno y entre 1 y 4 ampollas total de HMG (promedio 2.2 por ciclo por paciente), en la que ovularon. En las que no ovularon se alcanzó a administrar hasta 8 ampollas por ciclo, antes de abandonarlo.

No se encontró una mayor relación de LH/FSH en pacientes obesas. Sin embargo si hubo relación LH/FSH aumentada en las pacientes resistentes al esquema de inducción utilizado, lo que nos puede indicar que estos pacientes están en un estado avanzado de anovulación crónica.

Esto nos permite concluir que la obesidad per se si influye en la resistencia a la inducción de ovulación y que la relación LH/FSH alterada, que no es mayor en las obesas, también es un factor de mala respuesta.

El crecimiento folicular en las pacientes que van a terminar en ovulación se manifiesta desde temprano en el ciclo después de la terminación de la dosis de clomifeno y el comienzo de la administración de la gonadotrofina menopáusica. Por otra parte durante la administración de clomifeno el folículo no crece pero, inmediatamente después del pico esperado al día 5 de la administración del fármaco, que coincide con el inicio de HMG, comienza el crecimiento del folículo.

Cuando desde el día 10 del ciclo no se ve una buena progresión del tamaño folicular, el ciclo invariablemente terminará cancelado.

Con el esquema propuesto, se alcanza un buen porcentaje de ovulación en paciente que ha fallado el clomifeno, con disminución de costos y reducción de los efectos secundarios.

\section{Agradecimientos}

El suministro de HMG (Pergonal) y HCG (Profasi), fue gentilmente hecho por Laboratorios Farma Serono por intermedio del Dr. Hernán Briceño.

\section{BIBLIOGRAFIA}

1. Gómez G., Londoño RD., Cohen P. Inducción de ovulación: I clomifeno. Rev. Col. Obstet. Ginecol. 1986; 39: 409.

1. Sagle MA., Hamilton-Ferley D., Kiddy DS., Franks S. A comparative, randomized study of low-dose human menopausal gonadotropin and follicle-stimulating hormone in women with polycystic ovarian syndrome. Fertil Steril 1991; 55: 56.

2. Buvat J., Buvat H., Mercolin G., Dehaene JL., Verbecq P., Renovard O. Purified follicle stimulant hormone in polycystic ovary syndrome; slow administration is safer and more effective. Fertil Steril 1989; 52: 553.

3. Diugi A., Laufer N., Batero RW., Cherney A., Polann N., Hasiltine F., Mezer H., Bherman H. Alterd folicular development in clomiphene citrate versus human menopausal gonadotropin stimulated cycles for in vitro fertilization. Fertil Steril 1985; 43: 10.

4. Homburg R., Eshel A., Kilborn J., Adams J., Jacobs HS. Combined luteinizing hormone releasing hormone analogue and exogenous gonadotropins for the treatment of infertility associated with polycystics ovaries. Hum. Reprod. 1990; 5: 32.

5. Larsen T., Larsen JF., Shioler V., Bostofte E., Felding C. Comparison of urinary human follicle stimulant hormone and human menopausal gonadotropin for ovarian stimulation in polycystic ovarian syndrome. Fertil Steril 1990; 53: 426.

6. Dor J., Itzkovic DG., Mashiach S., Lunenfield B., Serr DM. Cumulative conception rate following gonadotropin therapy. Am. J. Obstet. Gynecol. 1980; 136: 102.
7. Remorgida V., Venturini P., Anserini P. Use of combined exogenous gonadotropins-releasing hormone in patients with polycystic ovarian disease. Fertil Steril 1991; 55: 61.

8. Marut EL., Hodgen G. Antiestrogenic action of high doses of clomiphene in primates pituitary segmentation but with ovarian attenuation. Fertil Steril 1982; 38: 102.

9. Yenn SSC. The polycystic ovary syndrome. Clin Endocrinol (oxf) 1980; 12: 177.

10. Silverberg K., Olive D., Burns W., Johnson J., Groff T., Schenken R. Follicular size at the time of human chorionic gonadotropin administration predicts ovulation outcome in human menopausal gonadotropin-stimulated cycles. Fertil Steril 1991; 56: 296.

11. Greemblatt RB., Barfield WE., Jungck EC., Ray AW. Induction of ovulation with MRL/41. JAMA 1961; 178: 101.

12. Gysler M., March CM., Mishell DR., Bailey EJ. A decade's experience with an individualized clomophene treatment regimen including its effect on the postcoital test. Fertil Steril 1982; 37: 161.

13. Diamond MP., Wentz AC. Ovulation induction with human menopausal gonadotropins. Obstetrical and Gynecological survey 1986; 41: 480.

14. Engel T., Jewelewicz R., Dyrenfurth I., Speroff L., Vande Wiele RL. Ovarian hyperstimulation syndrome: reporte of a case with notes on pathogenesis and treatment. Am. J Obstet. Gynecol. 1972; 112: 1052. 